\title{
On the "concentration-driven" methylene blue dimerization
}

\author{
P.A.R. Tafulo*, R.B. Queirós, G. González-Aguilar** \\ UOSE, INESC-Porto, Rua do Campo Alegre 687, Porto 4169-007, Portugal
}

\section{A R T I C L E I N F O}

\section{Article history:}

Received 11 August 2008

Received in revised form 10 February 2009

Accepted 19 February 2009

\section{Keywords:}

Methylene blue

UV-vis

Redox sensors

Dimerization

Optical sensors

\begin{abstract}
A B S T R A C T
In this work a theoretical and experimental analysis of the spectrum of methylene blue is made in order to clarify the nature of the shoulder appearing at $\sim 620 \mathrm{~nm}$. This shoulder has been several times attributed to the existence of an hypothetical methylene blue dimer. The results here obtained do not agree with the existence of such a dimeric specie, and point out differences in the ionic strength of the solution as the phenomenon responsible for the variations observed in these spectra.
\end{abstract}

(c) 2009 Elsevier B.V. All rights reserved.

\section{Introduction}

Methylene blue (MB) is a thiazine type dye, with interesting reversible redox properties that involve the equilibrium between the reduced (leuco) and the oxidized forms of this compound [1-4]. Because of that reversible equilibrium between the reduced and oxidized forms, $\mathrm{MB}$ is a compound useful as redox indicator [3,5-7]. Its main uses are related with the determination of glucose, $\mathrm{O}_{2}[5,8]$ or ascorbic acid [7] among others. The UV spectrum of MB has been widely studied $[9,10]$ in order to fully understand its chemical behavior.

Following several authors [9,10], the methylene blue dimerization equilibrium (represented in Fig. 1), can be expressed as $2 \mathrm{MB}_{\text {mon }} \rightleftharpoons \mathrm{MB}_{\text {dim }}$ and their equilibrium dimerization constant has the form,

$K_{d}=\frac{C_{d i m}}{C_{m o n}^{2}}$

as the free energy $(\Delta G)$ of a reaction is directly related with the equilibrium constant,

$\Delta G=-R T \ln K_{d}$

and the calculated constant for MB dimerization is around $10^{-3}$ $[9,10]$, then from the thermodynamic point of view $(\Delta G>0)$ the reaction is not favored.

* Corresponding author.

** Corresponding author. Tel.: +351933934170.

E-mail address: gaguilar@inescporto.pt (G. González-Aguilar).<smiles>CN(C)c1ccc2nc3ccc(=[N+](C)C)cc-3sc2c1</smiles>

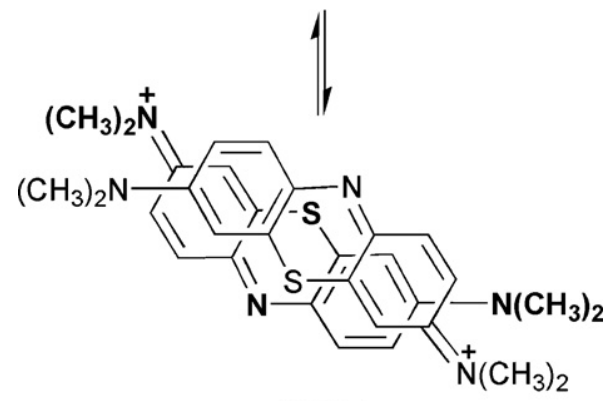

Dimer

Fig. 1. Chemical structure of methylene blue and its possible dimer.

The total concentration of $\mathrm{MB}$ can be calculated as $C=C_{\text {mon }}+$ $2 C_{d i m}$ and considering $x$ as the fraction of dye molecules existing in the monomeric form $\left(x=C_{m o n} / C\right)$, the equilibrium dimerization constant can be rewritten as

$K_{d}=\frac{1-x}{2 C x^{2}}$

this is a second degree equation that can be re-arranged in order to represent the values of the monomeric and dimeric species ( $x$ and $1-x)$ as a function of the total concentration $C$.

On the other hand, it is well known that the environment (solvents, other molecules) have strong influence in the UV spectra of a 


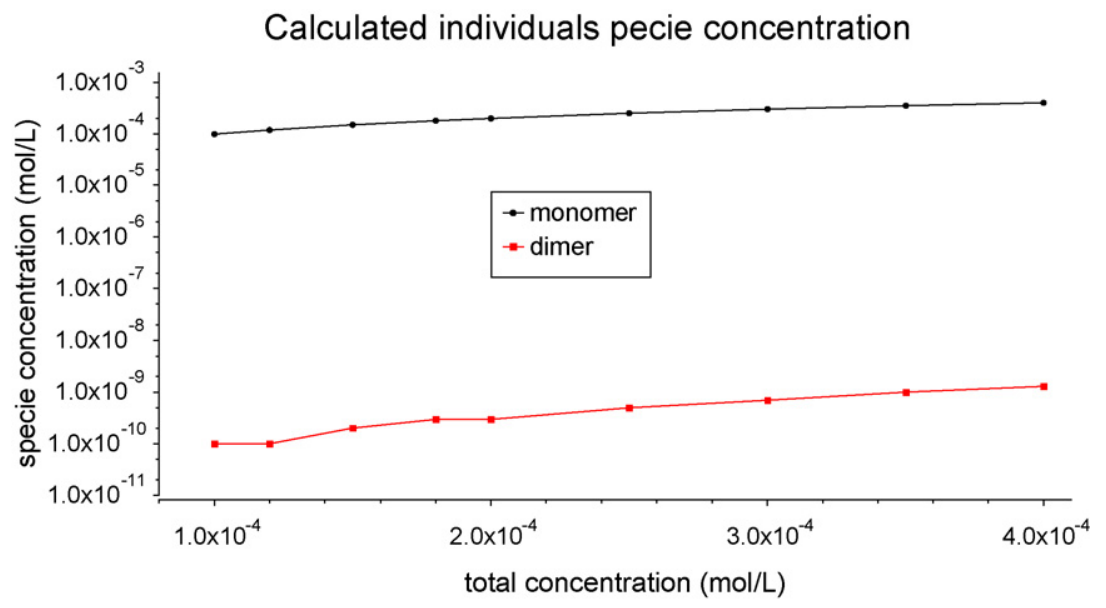

Fig. 2. Calculated concentration profiles for each specie.

compound. The interaction of the molecules with the environment has two important characteristics[11]:

\section{Transition bands is centered at different wavelength in solvents with} different polarities: as has been documented the increase of the polarity and the hydrogen bonding power of the solvent cause the shift of the $n-\pi^{*}$ bands to higher energies whereas the $\pi$ $\pi^{*}$ bands shifts to lower energies.
2. Bands suffer for inhomogeneous broadening due to fluctuations of the structure of the solvation shell surrounding the chromophore.

As a consequence of these two phenomena, it is unlikely that two bands due to different chemical species behave similarly in solvents of different polarities. It is expected that the equilibrium dimerization constant must be different when measured in dif-
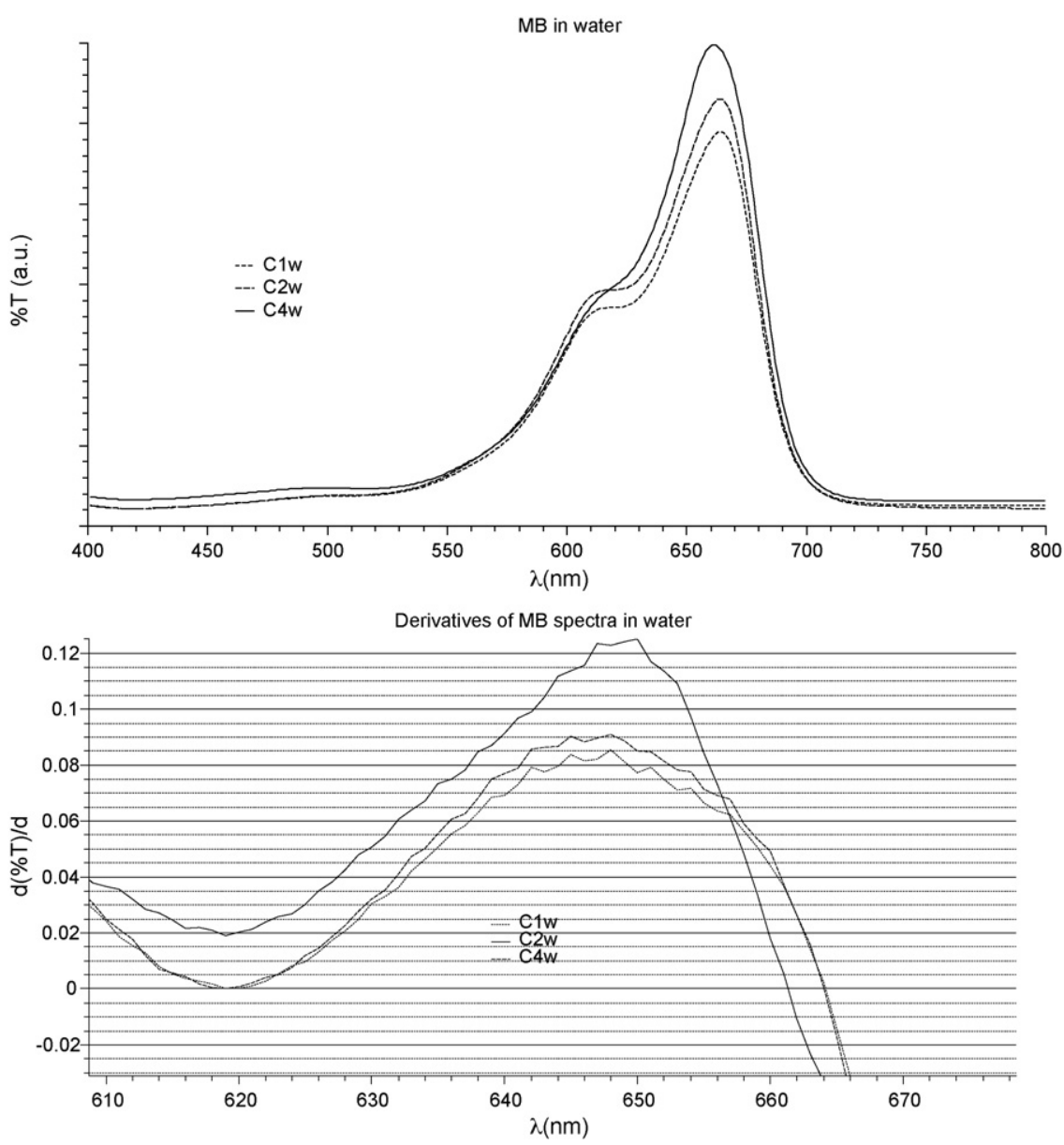

Fig. 3. UV-vis spectra of methylene blue in water (top) and its first derivative (bottom). 

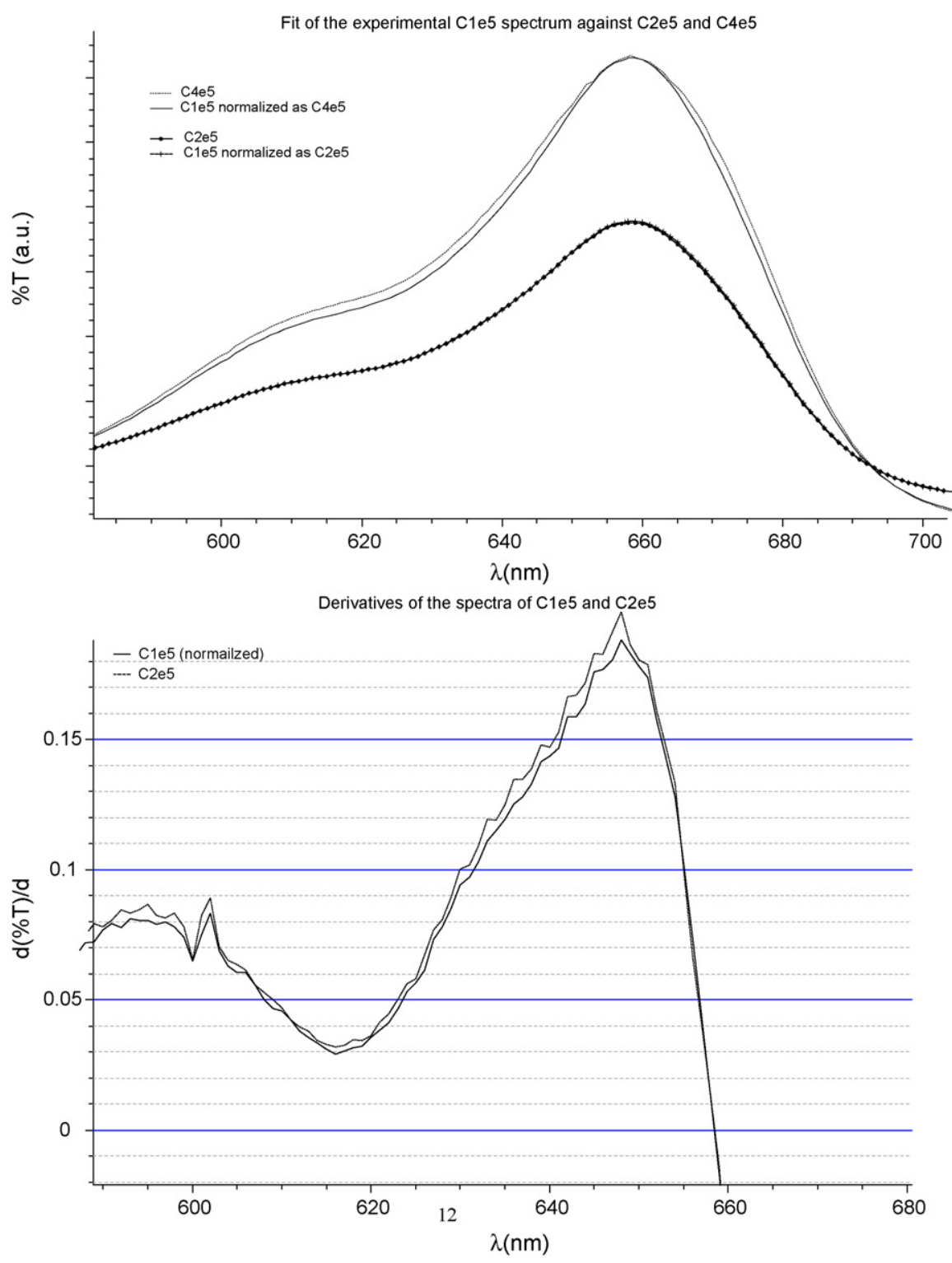

Fig. 4. UV-vis spectra of methylene blue in $\mathrm{EtOH}: \mathrm{H}_{2} \mathrm{O} 1: 1$ (top) and its first derivative (bottom).

ferent media and then the relative concentration of the dimeric and monomeric species must differ appreciably when measured in different environment. In this work theoretical modeling and practical measurements of the monomeric and dimeric band will be performed in order to access the electronic phenomena behind the $\sim 620 \mathrm{~nm}$ band clarifying whether it is or not correlated to the dimeric form of MB.

\section{Experimental}

Methylene blue (Riedel-de-Häen), ethanol (PanReac) and deionized water were used in all the experiments. Methylene blue $(0.01 \mathrm{~g})$ was dissolved in $25 \mathrm{ml}$ of deionized water to give a concentration of $1.25 \times 10^{-5} \mathrm{M}$. Aliquots $(0.1,0.2$ and $0.3 \mathrm{ml})$ of the above prepared solution were dissolved in $10 \mathrm{ml}$ of three different solvents: water and mixtures $1: 3$ and $1: 1$ parts of ethanol/water. The final concentration of the working solutions were $\left(1.25 \times 10^{-5}, 2.5 \times\right.$ $10^{-5}$ and $3.75 \times 10^{-5} \mathrm{M}$ ). The test solution is here addressed as $\mathrm{Cm} Y$ where $m$ is 1,2 or 4 and represents the concentrations $1.25 \times$ $10^{-5}, 2.5 \times 10^{-5}$ and $3.75 \times 10^{-5} \mathrm{M}$, respectively and $Y$ takes the value $w$ for water, $e 3$ for the mixture $1: 3$ of ethanol/water and $e 5$ for the mixture $1: 1$ of ethanol/water. As an example the sample $C 2 e 5$ is the sample with a methylene blue concentration of $2.5 \times 10^{-5} \mathrm{M}$ in a mixture $1: 1$ of ethanol/water. The UV-vis experiments were made with an Hitachi U-2010 spectrophotometer. Simulations and data analysis were carried out with the QTiPlot program [12].

\section{Results and discussion}

Fig. 2 shows the here calculated concentration profiles (using a re-arranged form of Eq. (3) of dimeric and monomeric MB at different total concentrations, following the described procedure. The calculated concentration of the dimer is always much lower than the corresponding concentration of the monomer. These results contradicts the values of $\varepsilon_{d}$ and $\varepsilon_{m}$ (both around $10^{4}$ ) reported by [9] and [10]) because as a consequence of the difference in concentrations between the monomeric and dimeric species the band associated with the dimeric species must be very low when compared with that associated to the monomeric specie. The quadratic order of Eq. (3) and the regression analysis demonstrates that the 

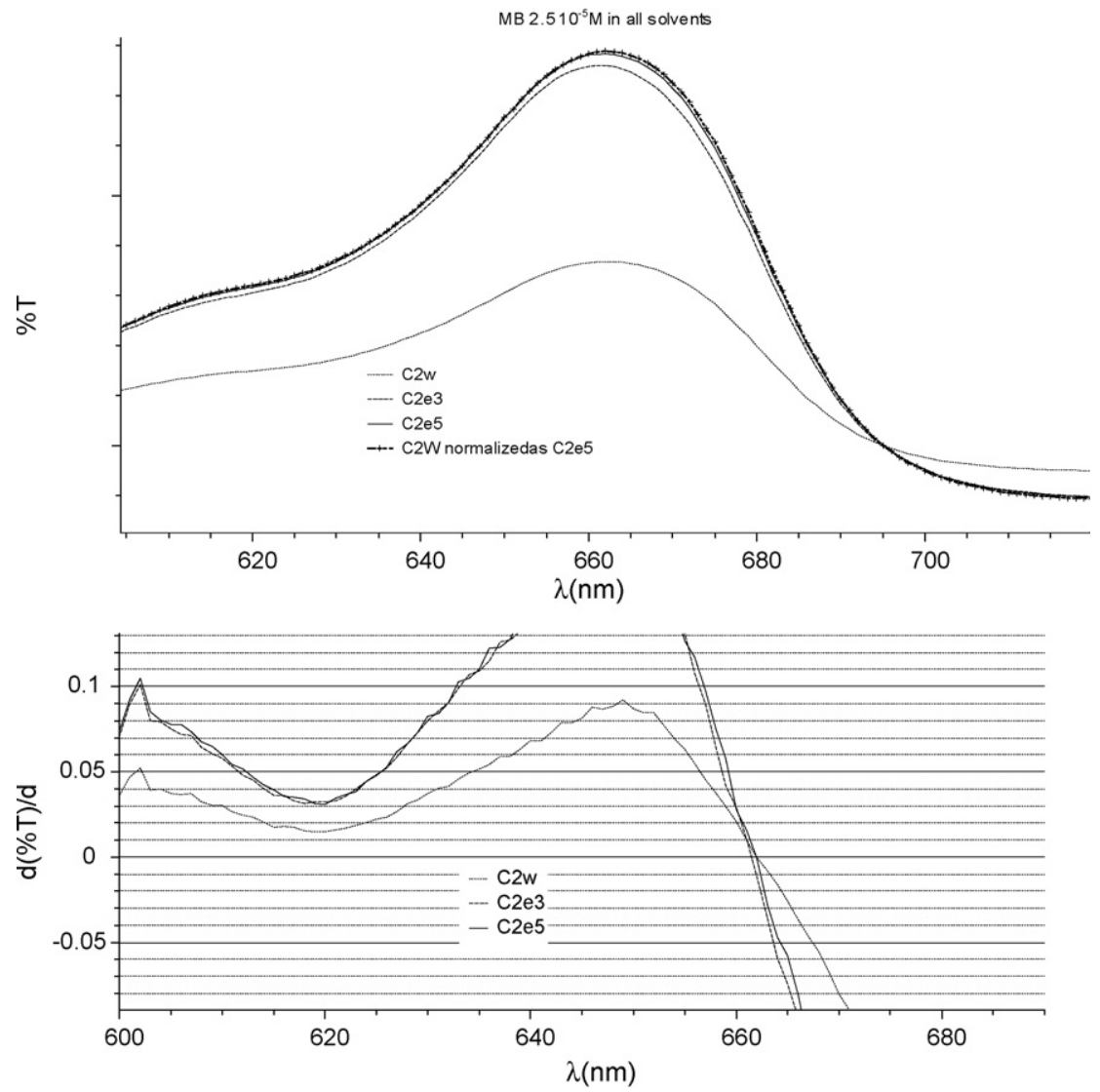

Fig. 5. UV-vis spectra of methylene blue $2.5 \times 10^{-5}$ in EtOH: $\mathrm{H}_{2} \mathrm{O} 1: 3$ (top) and its first derivative (bottom).

concentration of the monomeric specie grows linearly $(R=0.996)$ while the concentration of the dimeric specie is better described by a third degree equation $(R=0.976)$. Taking into consideration the differences in the concentrations between the two species it is clear that the only way the MB dimeric band can be noted in a normal spectrum is the case in which the absorption coefficient $\varepsilon_{d}$ of the dimeric specie is huge $\left(\sim 10^{4}\right.$ times $)$ when compared with that of the monomeric specie $\varepsilon_{m}$.

Fig. 3 shows the spectra (and its first derivatives) of MB solutions in water. A first impression denotes differences in the smaller band (at $\sim 618 \mathrm{~nm}$ ) of spectrum of the solution with the higher concentration (Fig. 3 top), but always the band situated at the higher wavelength is more intense than the band situated at the lower wavelength. These results agrees with those of Ghanadzadeh et al. (see Fig. 4 in [9]) but are contradictory with those reported by Patil et al. who have found that varying the concentration of MB causes a variation of the relative intensity of these bands (see Fig. 1 in [10]). A more detailed analysis of the characteristics of these bands at different concentrations can be made by using the derivative of the graph [13]. This analysis (Fig. 3 bottom) reveals that in the two more diluted solutions a shoulder is found $\left(f^{\prime}=0, f^{\prime \prime}=0\right)$, while in the more concentrated solution this shoulder disappears $\left(f^{\prime} \neq 0\right)$.

From these observations it is postulated the occurrence of bathochromic shift of this band when increasing the concentration of the solute. Moreover, from the obtained data at $\sim 655 \mathrm{~nm}$ it can be concluded that the solution obeys the Lambert-Beer Law,

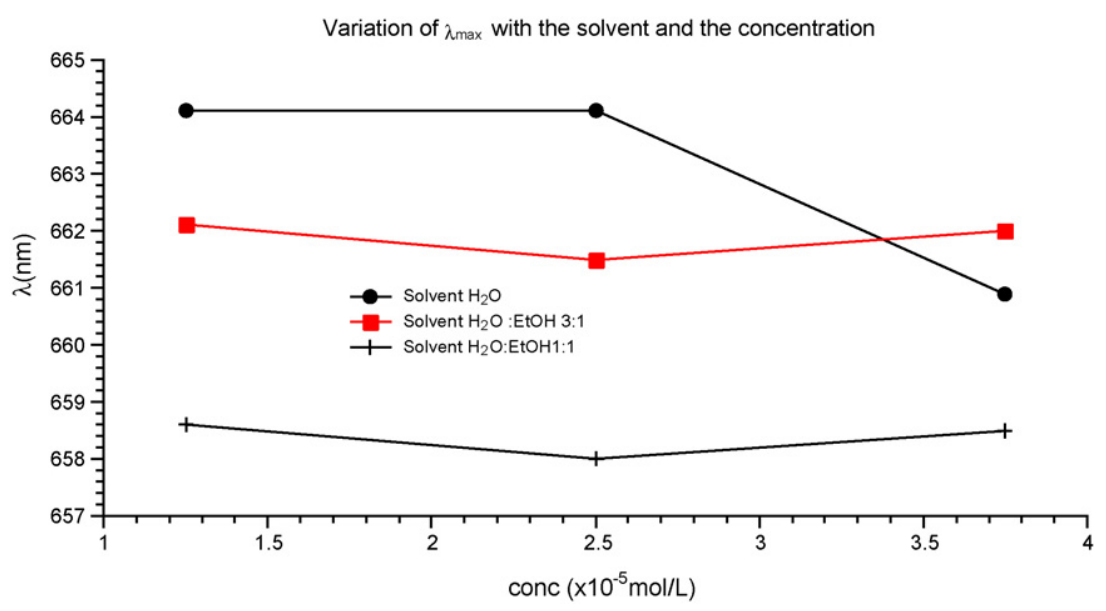

Fig. 6. Dependence of the intensity and position of $\lambda_{\max }$ with the concentration and the solvent. 


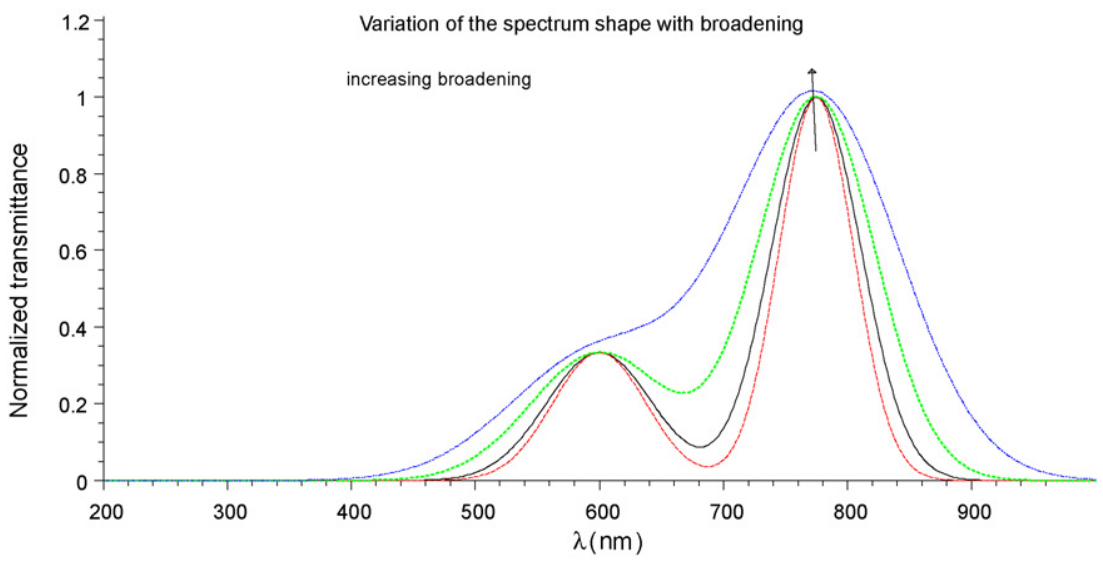

Variation of the spectrum shape with broadening and shift

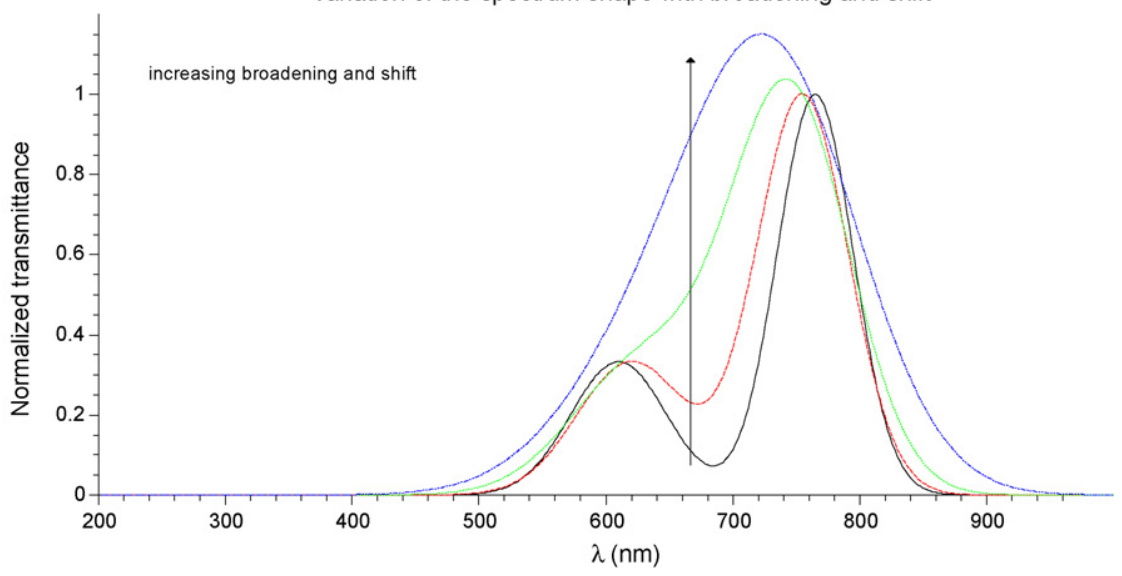

Fig. 7. Dependence of the overall shape of a spectrum with the broadening and the shift of the individual bands.

but the first derivative of the spectrum shows a little hypsochromic shift of this band depending of the concentration of the solution. In the case represented in that figure it is impossible to find an exact value for the position of the second band, as the band located at lower wavelength is joined to the one of lower energy.

The spectra of the C2e5 and C4e5 solutions are shown in Fig. 4(top). This figure also includes the normalized spectra of the less concentrated solution ( $C 1 e 5)$, taken the two more concentrated solutions as reference. The derivative of both spectra $C 2 e 5$ and $C 1 e 5$ (normalized as $22 e 5$ ) is showed in Fig. 4(bottom). From that figure, two important results arises:

- the spectra of the medium (C2e5) and lower ( $C 1 e 5)$ concentrated solutions are practically identical (when expressed one as function of the other), this means that in this case the surrounding media around the molecule is similar for both solutions.

- when normalizing the spectrum of the less concentrated solution (having $\mathrm{C} 4 \mathrm{e} 5$ one as reference), its bands are thinner than those bands of the $C 4 e 5$ solution, more over the band located at $\sim 615 \mathrm{~nm}$ growth less than the $665 \mathrm{~nm}$ band. In this case, the shift of the lower energetic band is not evident, because it can be masked by its broadening [11].

The influence of the different solvent on the position and shape of the bands of solutions with concentration $2.5 \times 10^{-6} \mathrm{M} \mathrm{MB}$ is shown in Fig. 5. In the same figure the normalized spectrum of $C 2 w$ against $C 2 e 5$ is represented. It is interesting to note that the spectra taken in different solvents seems to "obey" the Lambert-Beer Law, i.e. the normalization of them taking the other as basis make the spectra indistinguishable. Moreover, the intensity of the bands in the ethanolic solutions is always higher when compared with the water solutions of the same concentration and when increasing the ethanol content the intensity of the both bands (those assigned to the monomer and the dimer) also increases. This is a direct consequence of the different interactions of the solute with the solvent. These facts contradicts the proposed dimerization of MB. The dimerization constant is hardly of similar magnitude for solvents with different dielectric constants. However, accordingly with the Debye-Hückel double layer theory, each of these solutions have the same ionic strength [14] and then the broadening of the bands must be similar[11].

Fig. 6 up shows the variation of the position of the maximum of the spectra $\left(\lambda_{\max }\right)$ with the concentration for the MB solutions in the three studied solvents. As can be appreciated in that Figure, for the ethanolic solutions the position of $\lambda_{\max }$ remains almost constant (variation of $0.4 \mathrm{~nm}$ ). Oppositely, in the case of water $\lambda_{\max }$ diminish continuously but at higher concentrations the slope is of higher magnitude. It is noteworthy that only the case of the aqueous solution the Lambert-Beer behavior of the solutions at $\lambda_{\max }$ is valid. But on the other hand, the definition of the Lambert-Beer validates the use of the same wavelength for such measurements.

Some other considerations can help us to explain the so called linear growth of the "dimeric band". With this purpose here is modeled the theoretical absorption spectra based on the superposition of gaussians. For this purpose, it can be considered that the center of such gaussians is an indirect expression of the energy (position) of the band and the width indicating the influence of the environmental conditions. By altering these parameters, it is possible to 
represent the effect of the environment on both the position and broadening of the bands mentioned previously (see Section 1) and discussed by [11]. It is needed to remind here that a dilution of the sample to half the initial concentration means a lengthening of the distance between two adjacent molecules in the solute in a relation of $\sqrt[3]{2}$ (or $\sim 25 \%$ bigger) (see discussion about Debye-Hückel double layer theory in [14]) this change in the electrical field around ions in changing from a more diluted to a more concentrated solution can be related with the increase of the broadening of the bands on the above referred papers.

Fig. 7 (top) is a representation of the above described process (out of scale for clarity). The "intensity" $(I)$ of each individual band was simulated using the equation:

$I=\frac{e^{\left|\Delta x^{2} / B\right|}}{H}$

where $\Delta X$ is the distance from the center of the band, $B$ is a factor designing the broadening and $H$ is term related with the height of the band. The sum of the "intensity" of each band is then the calculated spectrum.

As can be seen a broadening of the bands promotes their fusion into a band and its shoulder, while a complementary shifting (Fig. 7, bottom) makes this process more pronounced. This process can be transposed to the case of the methylene blue molecule if we consider that the band at $660 \mathrm{~nm}$ is a $n-\pi^{*}$ transition which shifts to higher energies (lower $\lambda$ ) as the polarity of the medium increase with the concentration while at the same time the band at ( $\sim 620 \mathrm{~nm})$ (a $\pi-\pi^{*}$ band) moves to lower energies.

A situation such as describe before clarifies the variation of intensity the shoulder at $\sim 620 \mathrm{~nm}$ as being a process more related to the variation of the dielectric constant of the medium rather than being the change of the concentration of a hypothetical dimeric specie that contradicts the calculated profiles of concentration as demonstrated in Fig. 2.

\section{Conclusions}

The UV spectra of MB at several concentrations in solvents with different dielectric constants were studied. Surprisingly, the position and shape of the bands of this compound were very similar when dissolved in water, water/ethanol 3:1 or water ethanol 1:1. However in the water solution, the intensity of the bands was always lower when compared with those in ethanolic medium. The results here obtained do not point to the existence of a dimer as was referred by several authors. Moreover these results confirm the influence of the ionic strength of the solution on the position and shape of the bands as predicted by the Debye-Hückel theory. In that case it is likely than the band situated at $\sim 660 \mathrm{~nm}$ is a $n-\pi^{*}$ transition while those at $(\sim 620 \mathrm{~nm})$ is a $\pi-\pi^{*}$ one.

\section{References}

[1] K. Takahashi, M. Koitabashi, F. Kusu, Talanta 65 (2005) 1120-1125.

[2] M. Hepel, W. Janusz, Electrochim. Acta 45 (2000) 3785-3799.

[3] O. Impert, A. Katafias, P. Kita, A. Mills, A. Pietkiewicz-Graczyk, G. Wrzeszcz, J. Chem. Soc. Dalton Trans. (2003) 348-353.

[4] S.-K. Lee, A. Mills, J. Chem. Soc., Chem. Commun. (2003) 2366-2367.

[5] G.P. Gorbenko, Y.A. Domanov, J. Biol. Phys. Chem. 5 (1) (2005) 13-19.

[6] N. Tognalli, A. Fainstein, C. Vericat, M. Vela, R. Salvarezza, J. Phys. Chem. C 112 (2008) 3741-3746.

[7] Y. Dilgin, G. Nisli, Chem. Pharm. Bull. 53 (10) (2005) 1251-1254.

[8] L. Adamcikova, K. Pavlikova, P. Sevcik, Int. J. Chem. Kinetics 31 (6) (1999) 463-468.

[9] A. Ghanadzadeh, A. Zeini, A. Kashef, M. Moghadam, J. Mol. Liq. 138 (2008) 100-106.

[10] K. Patil, R. Pawar, P. Talap, Phys. Chem. Chem. Phys. 2 (2000) 4313-4317.

[11] B. Voleur, Molecular Fluorescence Principles and Applications, Wiley-VCH, Weinhelm, Germany, 2002

[12] Qtiplot, http://soft.proindependent.com/install.html.

[13] K. Jitmanee, J. Jakmunee, S. Lapanantnoppakhun, S. Wangkarn, N. Teshima, T. Sakai, G.D. Christian, K. Grudpan, Microchem. J. 86 (2007) 195-203.

[14] N. Lakshminayanaiah, Membrane Electrodes, Academic Press, NY, USA 1976. 